J. Electroanal. Chem., 336 (1992) 85-97

Elsevier Sequoia S.A., Lausanne

JEC 02174

\title{
Mass-transport-induced kinetic transitions during the electrochemical formation of three-dimensional dendritic silver deposits under ohmic control
}

\author{
A. Hernández Creus, P. Carro and S. González \\ Departamento de Química Física, Universidad de La Laguna, Tenerife (Spain)
}

\begin{abstract}
A.E. Bolzan, R.C. Salvarezza, S.L. Marchiano and A.J. Arvia
Instituto de Investigaciones Fisicoquímicas Teóricas y Aplicadas (INIFTA), Universidad Nacional de La Plata, La Plata (Argentina)
\end{abstract}

(Received 15 November 1991; in revised form 30 March 1992)

\begin{abstract}
The influence of mass transport processes on the growth of three-dimensional dendritic Ag deposits on a Pt quasi-spherical substrate at a relatively large cathodic overpotential has been investigated. After a certain induction time a first stage of dendritic growth of $\mathrm{Ag}$ can be observed. The deposit formed at this stage exhibits a fractal surface and non-fractal mass-volume properties. For charge densities greater than a certain critical value, a second stage of growth can be distinguished. At this second stage the contribution of free convection changes the shape of the deposit. The fastest growth of the $\mathrm{Ag}$ electrodeposit is determined by the rate of migration of sulphate ions in the electric field. A model accounting for the parametric relationships derived from the experiments is presented.
\end{abstract}

\section{INTRODUCTION}

The electrochemical formation of metal dendrites is a complex growth process. The influence of mass transport and ohmic drop in the electrolyte on the growth of rough solid surfaces deserves further investigation.

Under certain conditions of time and space the growth of metal dendrites, in either quasi-two-dimensional electrochemical cells or as a three-dimensional object, can be described by the development of fractals exhibiting characteristic Hausdorff dimensions [1-5]. The initiation of three-dimensional Ag dendrites exhibiting a fractal surface [6] requires a threshold charge related to an induction 
time. The fractal surface of the deposit was followed from the induction time up to a maximum time limited by the design of the experiment. These results pose several questions regarding changes in both the kinetics and the properties of the dendritic growth of $\mathrm{Ag}$, particularly for large electrodeposition charges.

The relatively high exchange current density of the $\mathrm{Ag} / \mathrm{Ag}^{+}$electrode reaction $\left(j_{0} \approx 1 \mathrm{~A} \mathrm{~cm}^{-2}\right)$ and the well-known kinetics of the $\mathrm{Ag} / \mathrm{Ag}^{+}$electrochemical system at high cathodic overvoltages make it suitable for studying the growth kinetics of $\mathrm{Ag}$ dendrites [7]. It was recently concluded that the rate of similar processes in $\mathrm{Cu}$ and $\mathrm{Zn}$ deposition is controlled by the rate of migration of anions in the solution [7-10].

This work investigates the growth of relatively large three-dimensional spongy $\mathrm{Ag}$ deposits through the electrodeposition of $\mathrm{Ag}^{+}$ion on a $\mathrm{Pt}$ substrate. It provides information about the following physicochemical aspects of the dendritic $\mathrm{Ag}$ deposits: (i) the fractal dimension of the surface and the non-fractal mass/volume of metal deposits; (ii) the contribution of the different mass transport processes in the rate of formation of $\mathrm{Ag}$ dendrites; (iii) the influence of the electric field in the kinetics of the process, a question which has recently been considered $[9,10]$ but it is not well understood for a three-dimensional growing object; (iv) a comprehensive description of dendritic $\mathrm{Ag}$ electrodeposits taking into account recent information reported on this process $[6,8]$.

\section{EXPERIMENTAL}

Several quasi-hemispherical Pt working electrodes facing axially downwards from the top of the electrochemical cell were used. The working electrode area covered the range $0.050-0.060 \mathrm{~cm}^{2}$. The charge density $q$ at time $t$ is the total charge deposited up to time $t$ divided by the area of the working electrode. The potential of the latter was measured against a saturated calomel electrode (SCE) placed in a separate compartment and connected to the rest of the cell through a fritted glass disc. The counter-electrode was a large-area Pt electrode.

It was proved that the entire cell design resulted in uniform radial diffusion and electric fields around the working electrode. Hence the initial instantaneous diffusion field around the electrode was calculated from the corresponding solution to Fick's law [11].

The dendritic Ag electrodeposits were grown at $25^{\circ} \mathrm{C}$ from $5 \times 10^{-3} \mathrm{M} \mathrm{Ag}_{2} \mathrm{SO}_{4}$ $+0.5 \mathrm{M} \mathrm{Na}_{2} \mathrm{SO}_{4}+0.01 \mathrm{M} \mathrm{H}_{2} \mathrm{SO}_{4}$ solution at a constant potential $E=-0.2$ $\mathrm{V} / \mathrm{SCE}$. The solution was prepared from p.a. reagents and Milli- $\mathrm{Q}^{*}$ water. Under these conditions, the morphology of the $\mathrm{Ag}$ dendrite could be ensured during a certain period of time [8]. All runs were made under an Ar atmosphere.

The change in the size of the growing object was followed sequentially by taking photographs at preselected electrodeposition charges. The photographs were taken with a Nikon FA camera coupled with a Micro Nikkor $55 \mathrm{~mm} \mathrm{f} / 2.8$ objective using a Ilford HP5 Plus film (ASA 400). In this way photographs of the growing deposits 
for values of $q$ up to $18 \mathrm{C} \mathrm{cm}^{-2}$ were obtained simultaneously with the charges passed as indicated by the coulometer.

Electrochemical measurements were made using PAR circuitry including model units 173, 175 and 179. Scanning electron micrographs were obtained using a Hitachi S-450 scanning electron microscope for beam energies ranging from 20 to $25 \mathrm{kV}$.

RESULTS

The shape of the growing object

A representative sequence of photographs corresponding to electrodeposited $\mathrm{Ag}$ dendrites (object) on Pt (substrate) is presented in Fig. 1. The growing object
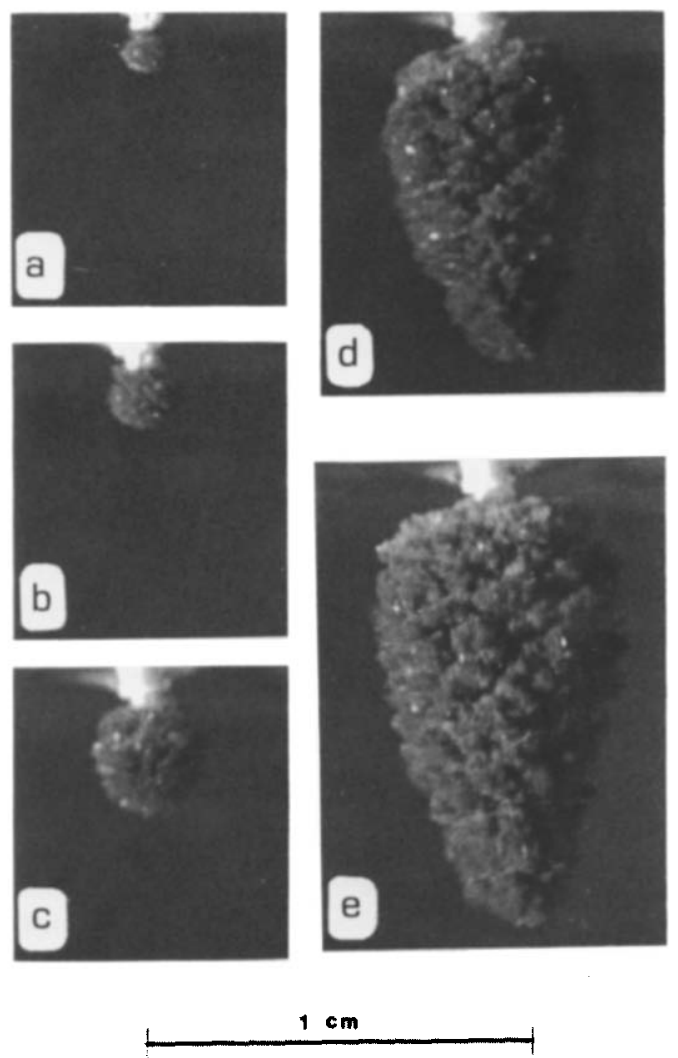

Fig. 1. Sequential photographs of the growing $\mathrm{Ag}$ dendrites obtained at different electrodeposition times. The corresponding values of $q$ are (a) $0.054 \mathrm{C} \mathrm{cm}^{-2}$, (b) $0.18 \mathrm{C} \mathrm{cm}^{-2}$, (c) $0.91 \mathrm{C} \mathrm{cm}^{-2}$, (d) $8.2 \mathrm{C}$ $\mathrm{cm}^{-2}$ and (e) $14.5 \mathrm{C} \mathrm{cm}^{-2}$. 

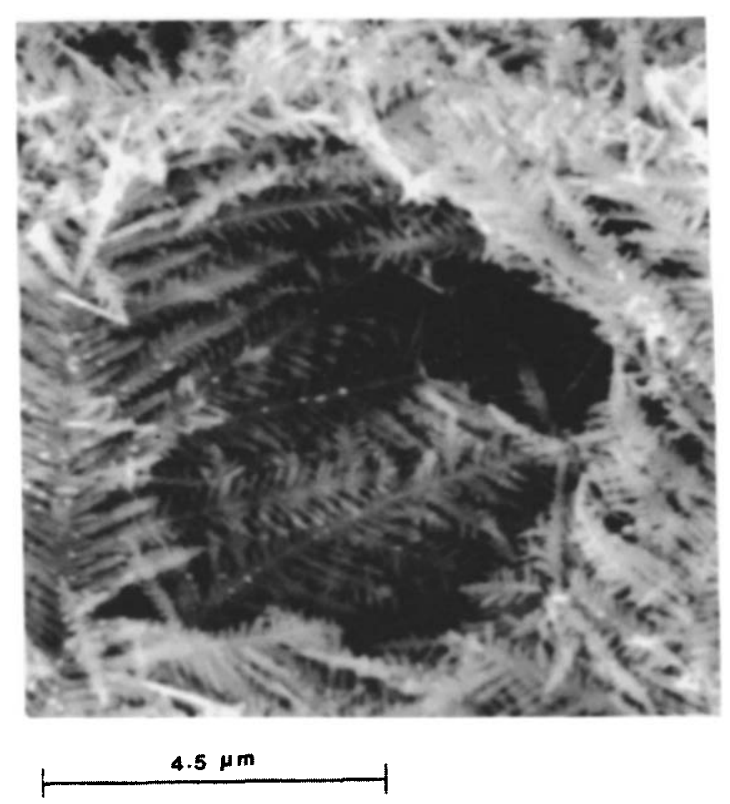

Fig. 2. Scanning electron micrograph of an $\mathrm{Ag}$ dendrite corresponding to $q=0.04 \mathrm{C} \mathrm{cm}^{-2}$. The bar scale is indicated. (Magnification $7000 \times$ ).

closely follows the shape of the substrate for values of $q$ up to about $0.18 \mathrm{C} \mathrm{cm}^{-2}$. When the value of $q$ exceeds $0.18 \mathrm{C} \mathrm{cm}^{-2}$, the geometry of the growing object changes from quasi-spherical to a "pine cone" shape. On the macroscopic level Ag dendritic deposits exhibit a sponge-like metallic structure, whereas ex-situ scanning electron micrographs at the micrometre level (Fig. 2) show that $\mathrm{Ag}$ deposits develop a "forest-like" display with a self-similar structure.

To determine the possible influence of the position of the working electrode on the shape of the object, similar experiments were performed with the substrate placed face upwards in the solution from the bottom of the cell. In these experiments a fungus-shaped $\mathrm{Ag}$ electrodeposit is formed.

\section{Phenomenological relationships}

A quantitative description of the dendritic growth of $\mathrm{Ag}$ can be accomplished by analysing different parametric relationships involving $q$, the geometric parameters of the object and time $t$. An axially symmetric object can be described in terms of the vertical length $L_{\mathrm{v}}$ and the maximum radial length $L_{\mathrm{h}}$ perpendicular to $L_{\mathrm{v}}$. In the present case these two parameters correspond to the principal growth directions of the object. The changes in both $L_{\mathrm{v}}$ and $L_{\mathrm{h}}$ during the growth process (Fig. 3) can be described as follows.

(i) $L_{\mathrm{v}}$ increases linearly with time throughout the experiment, with the slope of the straight line being $5.7 \times 10^{-4} \mathrm{~cm} \mathrm{~s}^{-3}$. 


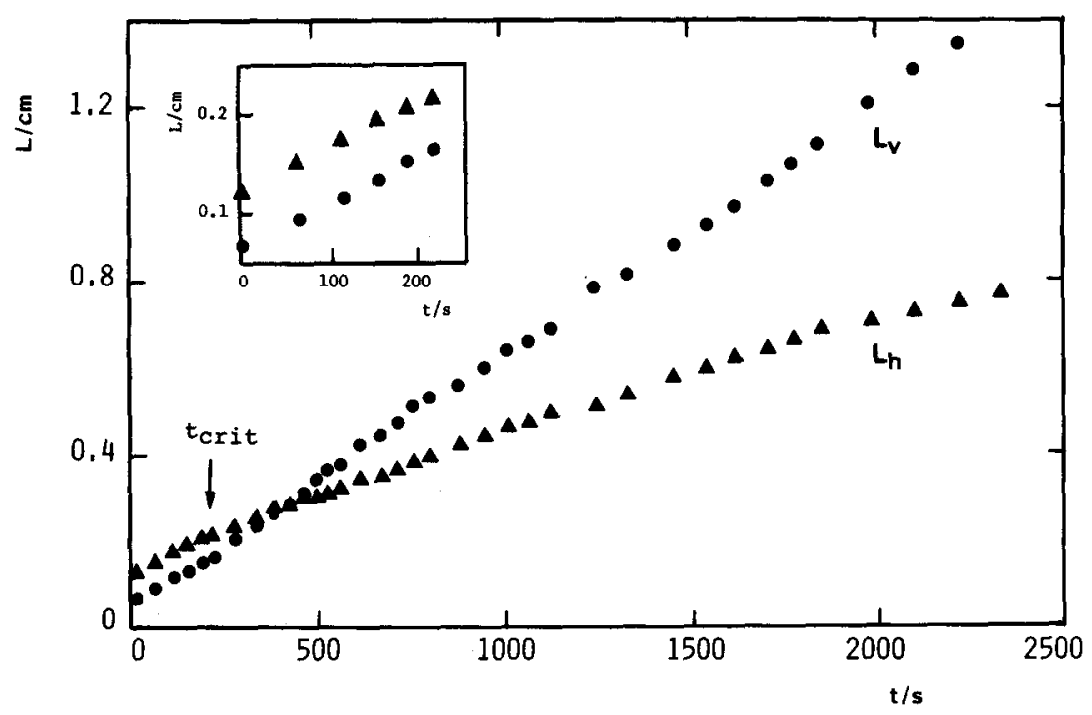

Fig. 3. Time dependence of $L_{\mathrm{v}}(\bullet)$ and $L_{\mathrm{h}}(\Delta)$. The initial portions of the plots are shown in the inset.

(ii) $L_{\mathrm{h}}$ increascs lincarly with time up to $q_{\text {crit }}=0.18 \mathrm{C} \mathrm{cm}^{-2}$ with the same slope as for $L_{\mathrm{v}}$.

(iii) When $q>q_{\text {crit }}$, the $L_{\mathrm{h}}$ vs. $t$ plot approaches a $L_{\mathrm{h}} \propto t^{x}$ power law with $x \approx 5 / 6$.

According to these results the initial isotropic growth of the object corresponds to the behaviour of a self-similar object (Figs. 1(a) and 1(b)), a conclusion which has been previously derived from scanning electron micrographs over a wide magnification range [6]. Otherwise, for $q>q_{\text {crit }}$ the growth of the object changes from isotropic to anisotropic with $\mathrm{d} L_{\mathrm{v}} / \mathrm{d} t>\mathrm{d} L_{\mathrm{h}} / \mathrm{d} t$ (Figs. 1(c)-1(e)).

Finally, the $q$ vs. $t$ plot results in a complex curve which does not fit a linear log $q$ vs. $\log t$ relationship (Fig. 4). This behaviour is due to the influence of the substrate geometry and of the appropriate growth laws of the object as discussed later.

\section{DISCUSSION}

The different stages in the dendritic growth of $\mathrm{Ag}$

The growth of the dendritic Ag electrodeposit implies the initial development of three-dimensional nuclei and the build-up of a diffusion layer around the substrate $[6,8]$. When a certain value of charge $q_{\mathrm{i}} \approx 0.018 \mathrm{C} \mathrm{cm}^{-2}$, which is related to the induction time $t_{\mathrm{i}}$ for dendritic growth, has passed, the thin Ag deposit begins to develop tips at the borders and angles of small crystals. These tips become larger than the thickness $\delta_{\mathrm{d}}$ of the diffusion layer resulting from Fick's law for a 


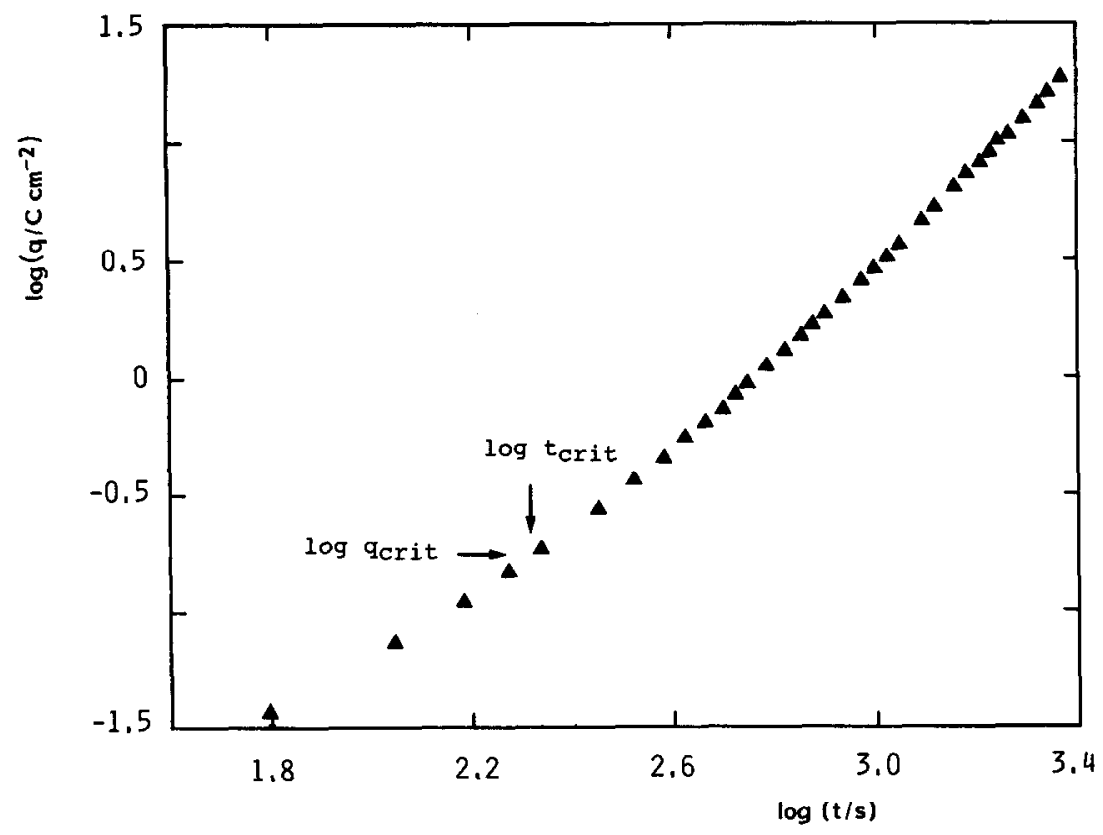

Fig. 4. Plot of $\log q$ vs. $\log t$ for the dendritic electrodeposition of $\mathrm{Ag}$.

hemispherical object. In this case, for a hemispherical electrode of radius $r \approx 0.1$ $\mathrm{cm}$, we find $\delta_{\mathrm{d}} \approx 0.05 \mathrm{~cm}$ at $t=t_{\mathrm{i}} \approx 30 \mathrm{~s}$ [12]. As the dendritic growth of $\mathrm{Ag}$ is triggered, the average size of the dendrites is already of the order of $0.1 \mathrm{~cm}$ (Fig. 3 ), i.e. a figure greater than $\delta_{\mathrm{d}}$. When $q_{\mathrm{i}}<q<q_{\text {crit }}$, the dendritic Ag deposit grows as an isotropic self-similar object.

When $q>q_{\text {crit }}$ anisotropic growth of the object begins to be observed (Fig. 3). At this stage, the size of the object becomes sufficiently large that hydrodynamic effects caused by density gradients in the solution produced during the electrode reaction begin to contribute to the kinetics of the process. The results obtained by changing the position of the working electrode also demonstrate that the development of free convection begins to play a significant role in the shape of the growing object. For the face-down position of the electrode this effect becomes evident for $q_{\text {crit }} \approx 0.18 \mathrm{C} \mathrm{cm}^{-2}$, which corresponds to $t_{\text {crit }} \approx 200 \mathrm{~s}$ (Figs. 3 and 4). The influence of free convection on the rate of dendritic growth of $\mathrm{Ag}$ is discussed later.

The fractal characteristics of the dendritic Ag deposit

To determine whether the Ag deposit behaves as a mass fractal object, the plot of mass $M$ vs. volume $V$ was investigated (Fig. 5). For this purpose the values of $M$ were obtained from the values of $q$ (Faraday's law) counted from $t=0$. The value of $V$ was calculated by assuming that the object can be modelled as a cylinder of 
height $L_{\mathrm{v}}$ and base diameter $L_{\mathrm{h}}$, and subtracting the volume of the substrate. The $M$ vs. $V$ plot can be drawn as a straight line covering almost three decades in both $q$ and $V$ (Fig. 5(a)). From the slope of this line, the apparent density $\rho$ of the object is found to be $1.66 \times 10^{-3} \mathrm{~g} \mathrm{~cm}^{-3}$, a figure which is much lower than the density of bulk $\mathrm{Ag}\left(\rho_{\mathrm{Ag}}=10.5 \mathrm{~g} \mathrm{~cm}^{-3}\right)$ and 1.5 times greater than the density of $\mathrm{Ag}^{+}$ions in the solution $\left(\rho=1.1 \times 10^{-3} \mathrm{~g} \mathrm{~cm}^{-3}\left(10^{-2} \mathrm{M} \mathrm{Ag}^{+}\right)\right)$. These results demonstrate that the dendritic $\mathrm{Ag}$ deposit is uniform with a mass dimension $D_{\mathrm{m}}=3$ over the entire growth range, i.e. it behaves as a mass non-fractal object. The low value of $\rho$ is consistent with the sponge-like aspect of the object and also with its terminal velocity in the electrolyte solution when the deposit is detached from the substrate [6].

Let us now consider the surface fractal characteristics of the deposit which has been observed for $q_{\mathrm{i}}<q<q_{\text {crit }}$ (Fig. 3). For a deposit which can be described as a self-similar object with $D_{\mathrm{m}}=3, A$ and $V$ are related by the equation

$A \propto V^{D_{\mathrm{s}} / 3}$

where $D_{\mathrm{s}}$ is the surface fractal dimension of the deposit. In this case $A$ was measured from the underpotential deposition of either $\mathrm{Pb}$ or $\mathrm{Cd}$, and $V$ was determined from the Ag electrodeposition charge $(V \propto q)$ [6]. The $\log A$ vs. $\log q$ plot (Fig. 5(b)), using data taken from ref. 6, shows a straight line with $D_{\mathrm{s}}=2.5 \pm$ 0.03 . Hence we can conclude from these results that the dendritic $\mathrm{Ag}$ deposit in the $q_{\mathrm{i}}<q<q_{\text {crit }}$ range, i.e. in the range of $q$ where eqn. (1) is valid, behaves as a surface fractal with non-fractal mass-volume properties.

Modelling the dendritic Ag electrodeposit

For an axially symmetric object growing on a quasi-hemispherical substrate of lengths $r_{0}$ and $2 r_{0}$ defined in the vertical and horizontal directions respectively, the instantaneous values of $L_{\mathrm{v}}$ and $I_{11}$ are given by

$L_{\mathrm{v}}=r_{0}+k_{\mathrm{v}} t^{y}$

and

$L_{\mathrm{h}}=2 r_{0}+k_{\mathrm{h}} t^{x}$

where $y$ and $x$ are the exponents of the growth law for $L_{\mathrm{y}}$ and $L_{\mathrm{h}}$ respectively, and $k_{\mathrm{v}}$ and $k_{\mathrm{h}}$ are constants. Accordingly, the effective instantaneous volume $V$, considering a cylinder, is

$V=V_{\mathrm{T}}-V_{0}=(\pi / 4)\left[L_{\mathrm{h}}^{2} L_{\mathrm{v}}-4 r_{0}^{3}\right]$

where $V_{\mathrm{T}}$ is the total instantaneous volume of the object and $V_{0}$ is the volume of the initial substrate. Taking into account the constant apparent density of the object, we can write (Fig. 4)

$$
\begin{aligned}
q \propto V= & (\pi / 4)\left(4 r_{0}^{2} k_{\mathrm{v}} t^{y}+4 r_{0}^{2} k_{\mathrm{h}} t^{x}+4 r_{\mathrm{v}} k_{\mathrm{h}} k_{\mathrm{v}} t^{(x+y)}\right. \\
& \left.+r_{0} k_{\mathrm{h}}^{2} t^{2 x}+k_{\mathrm{h}}^{2} k_{\mathrm{v}} t^{(2 x+y)}\right)
\end{aligned}
$$



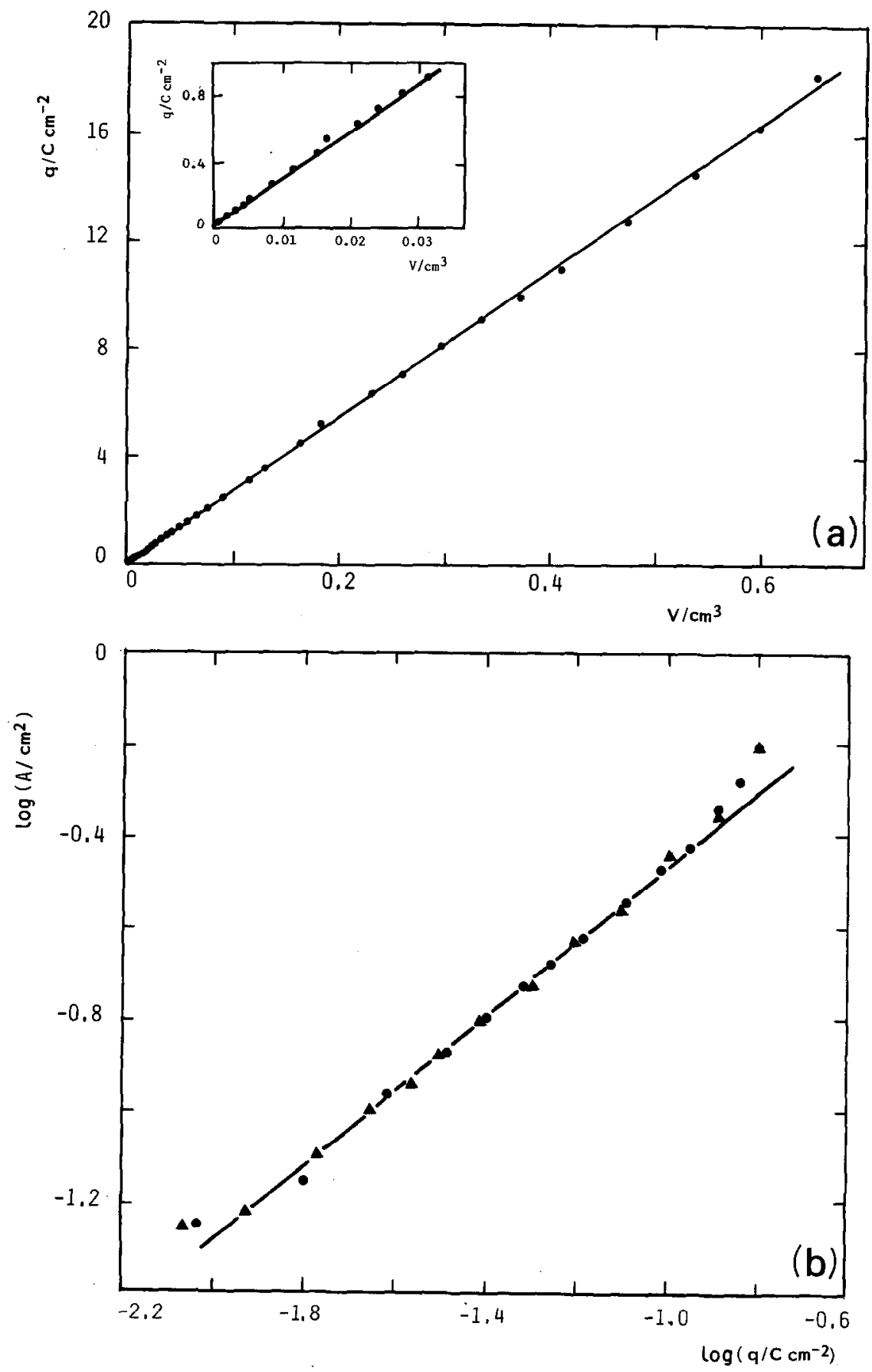

Fig. 5. (a) $q$ vs. $V$ plot (the initial portion of this plot is shown in the inset); (b) $\log A$ vs. $\log q$ p] the dendritic electrodeposition of $\mathrm{Ag}$ (data from ref. 6). 


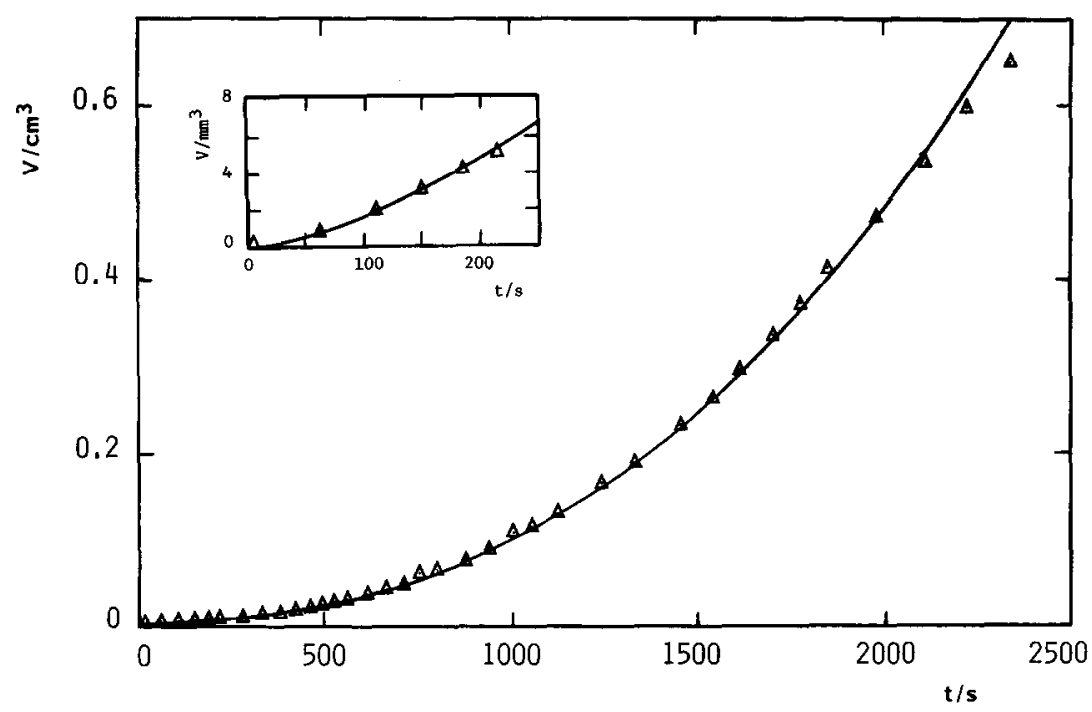

Fig. 6. $V$ vs. $t$ plot: $\Delta$, experimental data; - - , theoretical line calculated using eqn. (5). Experimental data and theoretical line resulting from eqn. (5) for $q<q_{\text {crit }}$ is shown in the inset.

This equation predicts a polynomial dependence of $q$ on $t$. According to eqn. (5) no linear relationships can be obtained in the $\log q$ vs. $\log t$ plot (Fig. 4). However, the validity of eqn. (5) extends to the entire range of the $q$ vs. $t$ plot provided that correct geometric parameters are taken. Thus for $q<q_{\text {crit }}$, i.e. in the range of isotropic growth, we can take $k_{\mathrm{h}}=k_{\mathrm{v}}=k$ and $x=y \approx 1$. Then eqn. (5) fits the initial part of the $V$ vs. $t$ plot (Fig. 6 , inset) by using $k=5.7 \times 10^{-4} \mathrm{~cm} \mathrm{~s}^{-1}$ (Fig. 3), $2 r_{0}=0.12 \mathrm{~cm}$ and $r_{0}=0.06 \mathrm{~cm}$.

For $q>q_{\text {crit }}$, when the anisotropic growth of the object becomes clearly visible, the following values are derived from the data in Fig. 3: $k_{\mathrm{h}}=1.15 \times 10^{-3} \mathrm{~cm}$ $\mathrm{s}^{-5 / 6}, k_{\mathrm{v}}=5.7 \times 10^{-4} \mathrm{~cm} \mathrm{~s}^{-1}, y=1$ and $x=5 / 6$. Using these values in eqn. (5), the experimental $V$ vs. $t$ plot can be entirely reproduced from $q_{\mathrm{i}}$ upwards (Fig. 6).

It should be noted that for $r_{0}=0$ and $k_{\mathrm{h}}=k_{\mathrm{v}}=k$, eqn. (5) becomes

$q \propto k^{3} t^{3}$

as predicted from computer simulations of three-dimensional growth at advanced stages [13].

Mass transfer contributions to the dendritic growth of $\mathrm{Ag}$

The electrochemical cell used in the present experiments can be discussed using an equivalent circuit involving a number of linear and non-linear resistors (Fig. 7). The resistances at the cathode (region $\mathrm{C}$ ) and the anode (region $\mathrm{A}$ ) are as follows: $\left(R_{\mathrm{e}}\right)_{\mathrm{A}}$ and $\left(R_{\mathrm{e}}\right)_{\mathrm{C}}$ associated with the electron-transfer reactions, the migration 


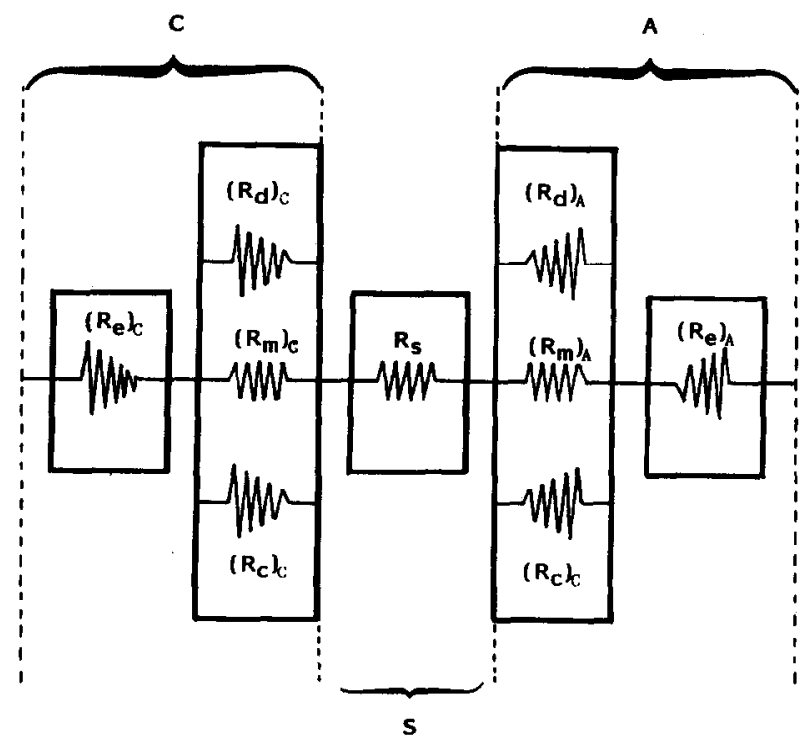

Fig. 7. Simplified equivalent circuit of the electrochemical cell.

contribution $\left(R_{\mathrm{m}}\right)_{\mathrm{A}}$ and $\left(R_{\mathrm{m}}\right)_{\mathrm{C}}$, the diffusion contribution $\left(R_{\mathrm{d}}\right)_{\mathrm{A}}$ and $\left(R_{\mathrm{d}}\right)_{\mathrm{C}}$, the convection contribution $\left(R_{\mathrm{c}}\right)_{\mathrm{A}}$ and $\left(R_{\mathrm{c}}\right)_{\mathrm{C}}$ to the transport of the reacting ions and the solution resistance $R_{\mathrm{s}}$ between the electrodes (region $\mathrm{S}$ ). In both regions $\mathrm{A}$ and $\mathrm{C}$ the values of $R_{\mathrm{m}}, R_{\mathrm{c}}$ and $R_{\mathrm{d}}$ can be represented as a parallel resistor arrangement in series with both $R_{\mathrm{e}}$ and $R_{\mathrm{s}}$. In the present case $R_{\mathrm{e}}$ is much smaller than the other resistive components, so that the kinetics of the electrodeposition reaction can be determined by either the rate of mass transport to the metal-solution interface or the ohmic resistance of the electrolyte solution. In addition, according to the experimental design we can further justify that those processes taking place in region $A$ are sufficiently fast that they can be disregarded in the analysis of the cathodic process.

At a single $\mathrm{Ag}$ dendrite tip $\delta_{\mathrm{d}}$ should become extremely small as, in principle, it is determined by the small radius of the dendrite tip $r_{\mathrm{t}} \approx 10^{-5}-10^{-6} \mathrm{~cm}$. Under these circumstances the value of $\delta_{\mathrm{d}}$ approaches the thickness $\delta_{\mathrm{DL}}$ of the diffuse double layer. Hence the concentration change of reacting ions takes place within a solution layer around the growing tip where the electroneutrality condition is no longer valid, so that the displacement of ions in this region should involve diffusion and migration contributions. This situation should be approached for $q<q_{\text {crit }}$.

The value of $t_{\text {crit }}$ (Fig. 3) can be related to the time required for establishing free-convection conditions around the object [11]. Accordingly, for $q>q_{\text {crit }}$ the change in the electrolyte concentration surrounding the growing object should produce a density gradient between the metal-solution interface and the bulk of the solution. This concentration change generates a macroscopic density gradient 
which produces free convection around the object [12]. Hence the onset of a convective cffect contributes to a further increase in the rate of $\mathrm{Ag}$ electrodeposition. The greater this effect, the thinner is the hydrodynamic layer. For an upward flow of solution impinging on the axially symmetric growing object the maximum effect of convection assisting the electrodeposition rate should occur at the impinging point, i.e. at the lowest point of the object. Accordingly, for $q>q_{\text {crit }}$ or $t>t_{\text {crit }}$ the contribution of convection produces a non-uniform rate of $\mathrm{Ag}$ electrodeposition along the surface of the object and correspondingly a change in the shape of the growing object. At this stage of deposit growth, diffusion, migration and free convection participate as mass transport contributions in region C (Fig. 7).

\section{Ohmic drop contributions}

As the rate of transport of reacting ions to the surface of the object becomes extremely large, a situation should be approached in which the movement of ions in the bulk of the solution determines the rate of the entire process. In this case the resistivity $R_{\mathrm{s}}$ of the solution should play a definite role in determining the kinetics of the global reaction. Under these conditions it would be expected that in the direction $L_{\mathrm{v}}$ where the fastest rate of deposit growth is attained, the rate equation for a two-dimensional growth process controlled by the anion migration holds [10]:

$v_{\mathrm{v}}=\mathrm{d} L_{\mathrm{v}} / \mathrm{d} t=-u_{-} E$

where $u_{-}$is the mobility of the anion and $E$ is the electric field in the vicinity of the "pine cone" tips. Although eqn. (7) is non-linear with respect to $t$, in practice $v_{\mathrm{y}}$ is constant in the thin cells [10,14]; this is also found in the present three-dimensional experiments (Fig. 3). Therefore according to this analysis one can conclude that the mobility of sulphate ions becomes rate determining in the $\mathrm{Ag}^{+}$ion transport process. As a matter of fact the maximum rate of dendritic growth of $\mathrm{Ag}$ obtained from Fig. 3 is $5.7 \times 10^{-4} \mathrm{~cm} \mathrm{~s}^{-1}$. This value lies within the range of maximum velocity expected for anion mobility in the solution which is of the order of $10^{-3}-10^{-4} \mathrm{~cm} \mathrm{~s}^{-1} \mathrm{~V}^{-1}$ [15]. Therefore in this case the growth rate of $\mathrm{Ag}$ dendrites is determined by the rate of migration of sulphate ions in the solution $[9,15]$.

A complete mechanistic description of the dendritic growth of $\mathrm{Ag}$

The preceding discussion, together with previously reported data concerning the early stages of the electrodeposition of $\mathrm{Ag}$ under the same experimental conditions, allows us to give a complete mechanistic description of the electrochemical growth of $\mathrm{Ag}$ dendrites at high negative overvoltages. This mechanistic description can be outlined as follows.

(i) The initial deposition of $\mathrm{Ag}\left(q<q_{\mathrm{i}}\right)$ at a relatively large negative overpotential $(E=-0.200 \mathrm{~V})$ corresponds to an electrochemical diffusion-controlled $\mathrm{Ag}^{+}$ 
ion discharge producing well-defined small crystals whose average sizes are less than the average thickness of the diffusion layer built up around the substrate electrode $[6,8]$.

(ii) When $q_{\mathrm{i}}<q<q_{\text {crit }}$ dendritic growth of $\mathrm{Ag}$ takes place. The deposit evolves isotropically, leading to self-similar objects with $D_{\mathrm{m}}=3$ and $D_{\mathrm{s}}=2.5 \pm 0.03$. There are simultaneous large migration and diffusion contributions to the electrochemical reaction.

(iii) When $q_{\text {crit }}$ is exceeded, the contribution of free convection to the transport of reacting ions commences and anisotropic growth of the object prevails. Then the rate of $\mathrm{Ag}$ electrodeposition depends on the geometric parameters of the object, with the largest rate appearing at the lowest part where the upward solution stream impinges on the object and coincides with the mobility of sulphate ions in the electrolyte solution.

(iv) The entire dendritic growth process of $\mathrm{Ag}$ under the present conditions can be described as an electrochemical formation of a new phase influenced by migration, diffusion and convection under ohmic control.

(v) The relative extent of the mass transport contribution to the total electrodeposition process depends on a number of variables such as the cathode potential, the composition of the solution, the physicochemical characteristics and geometry of the working electrode, the cell design and the temperature. The influence of several of these variables has been already reported elsewhere $[6,8]$.

\section{ACKNOWLEDGEMENTS}

This work was developed within the framework of the Programa de Cooperación Científica con Iberoamérica (1991) de los Ministerios de Educación y Ciencia y de Asuntos Exteriores of Spain. The authors thank the referee for helpful suggestions and comments on the preparation of the revised manuscript.

\section{REFERENCES}

1 T. Vicsek, Fractal Growth Phenomena, World Scientific, London, 1989.

2 C.P. Chen and J. Jorné, J. Electrochem. Soc., 137 (1990) 2047.

3 P.P. Trigueros, J. Claret, F. Mas and F. Sagués, J. Electroanal. Chem., 312 (1991) 219.

4 T.A. Witten and L.M. Sander, Phys. Rev. Lett., 47 (1981) 1400.

5 L.M. Sander, Sci. Am., 256 (1987) 94.

6 A. Hernández Creus, P. Carro, S. González, R.C. Salvarezza and A.J. Arvia, J. Electrochem. Soc., 139 (1992) 1064.

7 G. Hills, A.K. Pour and B. Scharifker, Electrochim. Acta, 28 (1983) 891.

8 A. Hernández Creus, P. Carro, S. González, R.C. Salvarezza and A.J. Arvia, Electrochim. Acta, in press.

9 V. Fleury, J.N. Chazalviel, M. Rosso and B. Sapoval, J. Electroanal. Chem., 290 (1990) 249.

10 J.R. Melrose, D.B. Hibert and R.C. Ball, Phys. Rev. Lett., 65 (1990) 3009.

11 P. Delahay, New Instrumental Methods in Electrochemistry, Interscience, London, 1954.

12 B. Levich, Physicochemical Hydrodynamics, Prentice-Hall, Englewood Cliffs, NJ, 1962.

13 P. Meakin and J.M. Deutch, J. Chem. Phys., 80 (1984) 2115. 
14 P. Garik, D. Barkey, E. Ben-Jacob, E. Bochner, N. Broxholm, B. Miller, B. Orr and R. Zamir, Phys. Rev. Lett., 62 (1989) 2703.

15 T. Erdey-Gruz, Transport Phenomena in Aqueous Solutions, Adam Hilger, London, 1974, Chapter 4, p. 253. 